\title{
LES PHLÉBOTOMES IMPLIQUÉS DANS LA TRANSMISSION DES LEISHMANIOSES DANS L'ILE DE GOZO (MALTE).
}

\author{
N. LÉGER*, R. MARCHAIS*, G. MADULO-LEBLOND**, B. PESSON***, A. KRISTENSEN***, \\ H. FERTÉ*, R. KILLICK-KENDRICK****, M. KILLICK-KENDRICK****
}

RÉSUMÉ

$\mathrm{Au}$ cours d'une enquête dans le foyer leishmanien de Gozo (Malte) plus de 16000 phlébotomes ont été capturés par divers procédés et 6 espèces répertoriées : Sergentomyia minuta, Phlebotomus papatasi, $P$. sergenti, $P$. neglectus, $P$. perfiliewi et $P$. per- niciosus. Cette dernière espèce, trouvée infestée par Leishmania infantum, a fait l'objet d'une étude isoenzymatique qui n'a révélé que des différences minimes avec d'autres populations de la même espèce en provenance d'Espagne et de France.

Summary: Study of the sandflies, vectors of leishmaniasis in the island of Gozo (Malta).

The sandflies of Gozo, a focus of leishmaniasis, have been investigated More than 16000 sandflies have been catched by various means. They belong to 6 species: Sergentomyia minuta, Phlebotomus papatasi, $P$. sergenti, $P$. neglectus, $P$. perfiliewi and $P$. perniciosus. P. perniciosus which has been found infected with Leishmania infantum, has been studied for izoenzymes and found nearly identical to other populations originated from Spain and France.

\section{INTRODUCTION}

Les îles maltaises situées au cœur du Bassin Méditerranéen sont un foyer de leishmaniose viscérale connu de longue date.

Entre 1946 et 1984, 1185 cas ont été répertoriés pour les deux îles de Malte et de Gozo (Vella Briffa, 1985) et 38 de 1984 à 1988 soit 37 à Malte (318 334 habitants en 1986) et 1 à Gozo (25 112 habitants) (Ministère de la Santé de Malte, 1989).

En revanche, si l'on exclut quelques cas de lésions cutanées post kala-azar, la leishmaniose cutanée proprement dite semblait absente des deux îles lorsqu'en 1981 une touriste anglaise contracta un «bouton d'Orient» à Gozo. Alertés par ce premier cas, les dermatologistes maltais en diagnostiquèrent 13 autres entre février 1982 et février 1985 , tous en provenance de l'île de Gozo (Vella Briffa, 1985).

Le problème qui se posait alors était de savoir si Leishmania tropica était effectivement présente à Gozo ou si les lésions cutanées observées devaient être rapportées à L. infantum comme cela est le cas dans diverses régions méditerranéennes (Rioux et coll., 1980). La solution pas-

* Faculté de Pharmacie de Reims, F 51096 Reims cedex.

** Faculté de Pharmacie de Paris XI, F 92290 ChatenayMalabry.

*** Faculté de Pharmacie de Strasbourg, F 67048 Illkich cedex.

**** Department of Pure and Applied Biology, Imperial College at Silwood Park Ascot, Berkshire SL5 7DE UK.

Accepté le : 11 mars 1991. sait non seulement par l'isolement et le typage des parasites à partir de lésions humaines, mais aussi par l'individualisation des vecteurs.

Les phlébotomes de Gozo n'avaient pas été étudiés. En revanche, 6 espèces étaient connues de l'île de Malte : Phlebotomus papatasi, Phlebotomus perniciosus, Sergentomyia minuta (Newstead, 1911 ; Marett, 1943), Phlebotomus sergenti, $P$. neglectus (=P. major) et $P$. perfiliewi (=P. macedonicus) (Adler et Théodor, 1935).

L'espèce Phlebotomus nigerrimus signalée et décrite par Newstead en 1911 devait dès 1914 être mise en synonymie avec $P$. perniciosus par l'auteur lui-même.

Comme le faisait déjà remarquer Théodor en 1975, le rôle vecteur de $P$. papatasi évoqué par Cachia et Fenech (1964) et Busuttil (1974) semblait peu probable compte tenu de ce qu'on connaît actuellement de la spécificité parasitaire chez les phlébotomes. Le vecteur le plus vraisemblable était l'un des trois Larroussius ( $P$. perniciosus, principal suspect en raison de son abondance, $P$. neglectus et $P$. perfiliewi) pour Leishmania infantum et $P$. sergenti pour L. tropica. L'isolement de leishmanies à partir de l'une ou l'autre de ces espèces constituerait un des éléments de la réponse à la question posée.

Une enquête épidémiologique débute en août 1988 à Gozo. Elle a pour but :

- d'isoler des souches de leishmanies en vue de leur typage enzymatique afin de déterminer s'il s'agit uniquement de souches dermotropes de $L$. infantum ou si $L$. tropica est également présente dans l'île, 


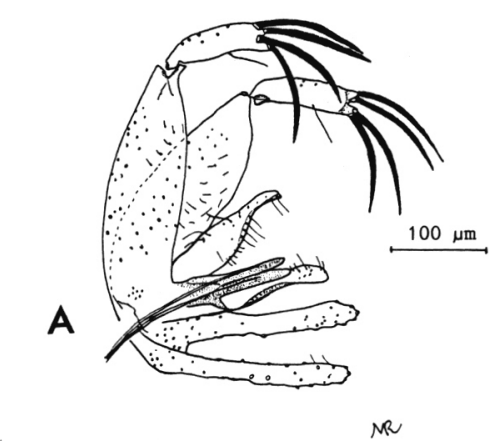

SERGENTOMYIA MINUTA
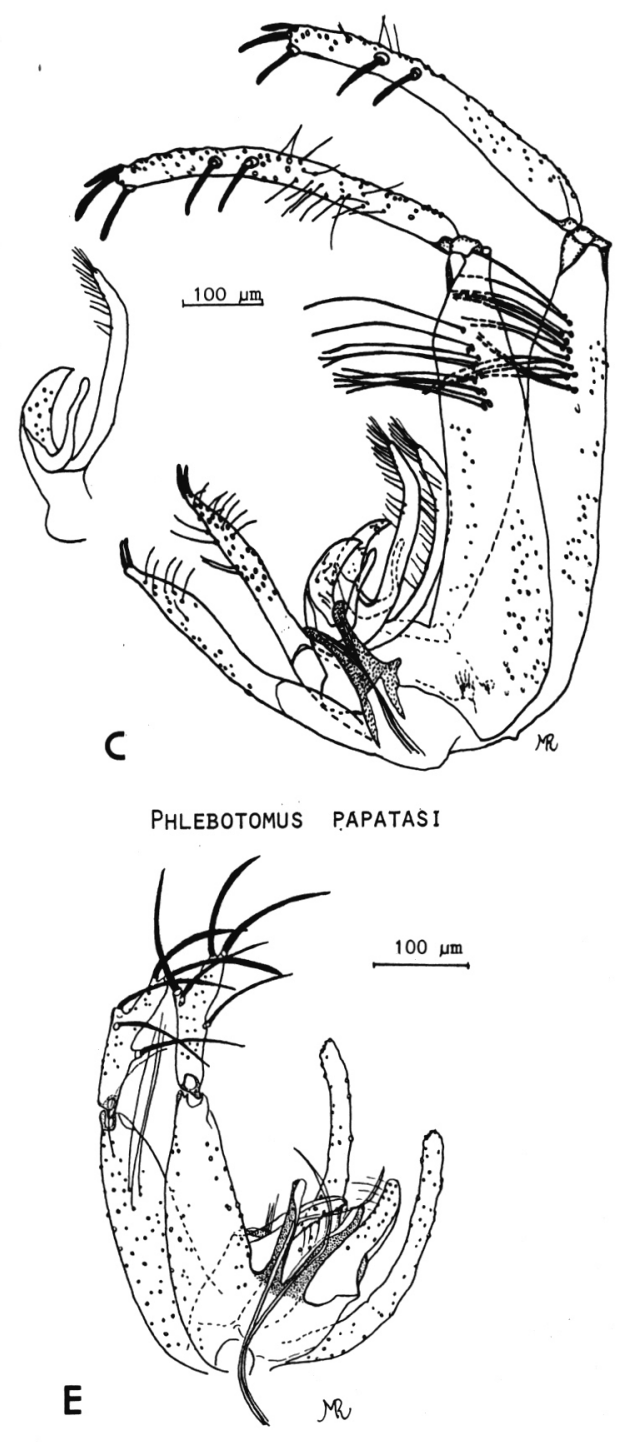

PhLebotomus Perfiliewi
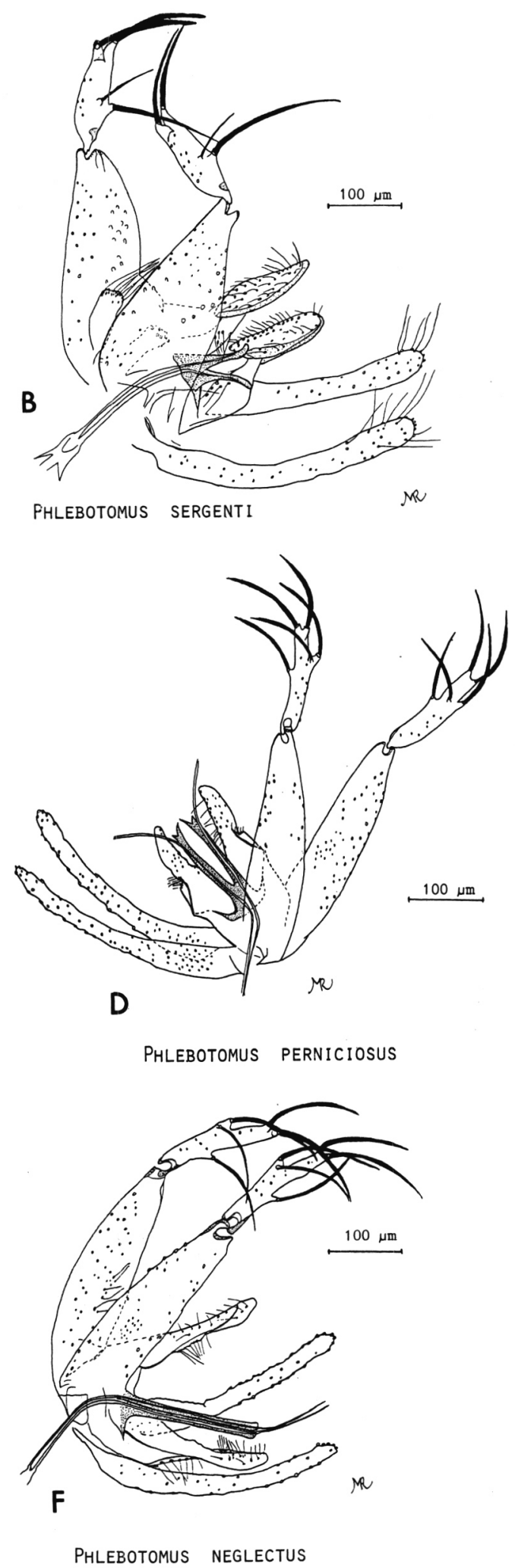

FIG. 1. - Genitalia mâles des six espèces de phlébotomes retrouvées à Gozo (Malte). 
- d'identifier le ou les vecteurs responsables,

- de rechercher les réservoirs de virus et de déterminer leur degré d'infestation.

Le présent article est consacré à l'enquête entomologique qui s'est déroulée en 1988 (20 août-3 septembre) et en 1989 (25 juin-23 juillet).

\section{MÉTHODES}

1 - PiéGeage

Afin de disposer d'un matériel d'étude représentatif à la fois qualitativement et quantitativement, différentes méthodes d'échantillonnage ont été utilisées :

— pièges adhésifs à l'huile de ricin disposés le long d'itinéraires transects (Croset et coll., 1977),

- pièges lumineux de type C. D. C. miniature, modifiés par R. Killick-Kendrick,

- captures manuelles nocturnes sur mur ou sur appât humain.

2 - OBSERVATION ET IDENTIFICATION

A - pour les mâles : montage permanent dans le baume du Canada,

B - pour les femelles : dissection des spermathèques, éventuellement après isolement des tubes digestifs (cf. infra) et montage dans la gomme au chloral (Léger et coll., 1983).

3 - Isolement des leishmanies à partir des femelles capturées vivantes : dissection dans le tampon $P$. B. S. stérile et le cas échéant culture sur milieu d'Evans (Peters et KillickKendrick, 1987).

4 - Conservation dans l'azote liquide des mâles capturés vivants.

5 - Mise en élevage des femelles gravides ou gorgées.

\section{RÉSULTATS}

\section{A - INVENTAIRE FAUNistiQue}

1 - LISTE DES ESPÈCES TROUVÉES ET CLÉ DE DÉTERMINATION

$\mathrm{Au}$ cours de cette enquête, six espèces de phlébotomes ont été trouvées :

- genre Sergentomyia:

Sergentomyia minuta (Rondani, 1843).

- genre Phlebotomus :

Sous-genre Phlebotomus :

Phlebotomus papatasi (Scopoli, 1786).

Sous-genre Paraphlebotomus :

Phlebotomus sergenti Parrot, 1917.

Sous-genre Larroussius :

Phlebotomus neglectus Tonnoir, 1921.

Phlebotomus perfiliewi Parrot, 1930.

Phlebotomus perniciosus Newstead, 1911.
Clé de détermination

- Présence d'un appareil copulateur externe : mâle

1 - Style avec 4 épines terminales +1 soie caduque médiane; pas de processus sur le coxite :

Sergentomyia minuta (fig. $1 \mathrm{~A}$ ).

2 - Style avec 4 épines dont 2 terminales +2 médianes dont l'une est souvent de taille réduite; un processus basal sur le coxite, garni de longues soies :

Phlebotomus sergenti (fig. 1B).

3 - Style avec 5 épines :

- Paramère trifurqué :

Phlebotomus papatasi (fig. 1C).

. Paramère simple :

- Valves péniennes longues, en baguette de tambour :

Phlebotomus neglectus (fig. 1F).

- Valves péniennes spatulées à leur extrémité : Phlebotomus perfiliewi (fig. 1E).

- Valves péniennes bifurquées à leur extrémité : Phlebotomus perniciosus (fig. 1D).

- Pas d'appareil copulateur externe: femelle

1 - Cibarium armé et fortement pigmenté, spermathèques lisses (non annelées) :

Sergentomyia minuta (fig. 2A).

2 - Cibarium inerme, spermathèques annelées :

a - tête de la spermathèque sessile :

. corps de la spermathèque formé de 4 à 7 anneaux, le dernier dans lequel la tête est invaginée est plus haut que les autres. Pharynx orné de fortes écailles disposées en réseau :

Phlebotomus sergenti (fig. 2B);

. corps de la spermathèque formé de 8 à 10 anneaux de même taille; tête en " pompon ". Pharynx recouvert d'écailles finement dentelées :

Phlebotomus papatasi;

$\mathrm{b}$ - tête de la spermathèque portée par un long cou :

. un conduit commun :

Phlebotomus neglectus (fig. 2C)

- pas de conduit commun mais des dilatations de forme caractéristique à la base de chaque conduit : Phlebotomus perfiliewi (fig. 2D),

Phlebotomus perniciosus (fig. 2E).

2 - RÉsultats QUanTitatifs

1 - Études chorologiques par la technique des pièges adhésifs, le long d'un itinéraire transect (carte 1) :

. une le 22 août 1988 (pièges relevés le 25 ) : 370 papiers posés (311 récupérés, soit une moyenne de 20,7 par station). 6808 phlébotomes ont été capturés, soit une moyenne de 2189 pour 100 papiers.

. l'autre les 12-13 juillet 1989 (pièges relevés le 16-17) : 351 papiers posés ( 327 récupérés, soit une moyenne de 20,4 


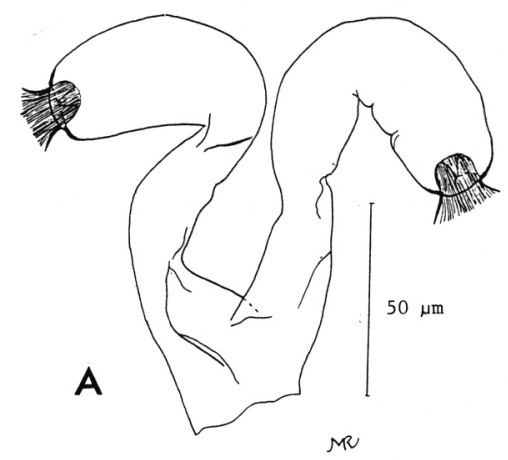

SERGENTOMYIA MINUTA
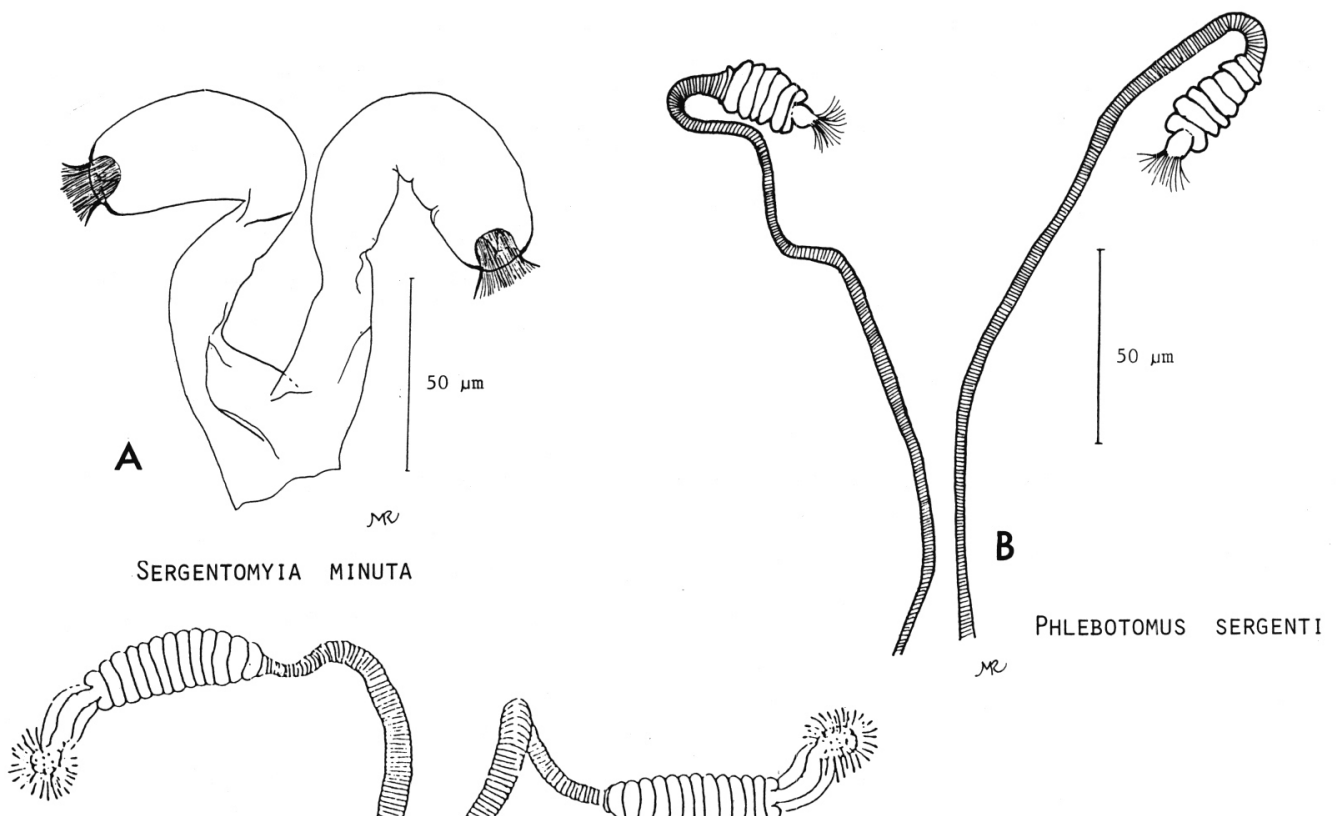

C

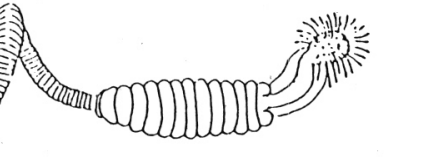

PHLEBotomus NegLectus

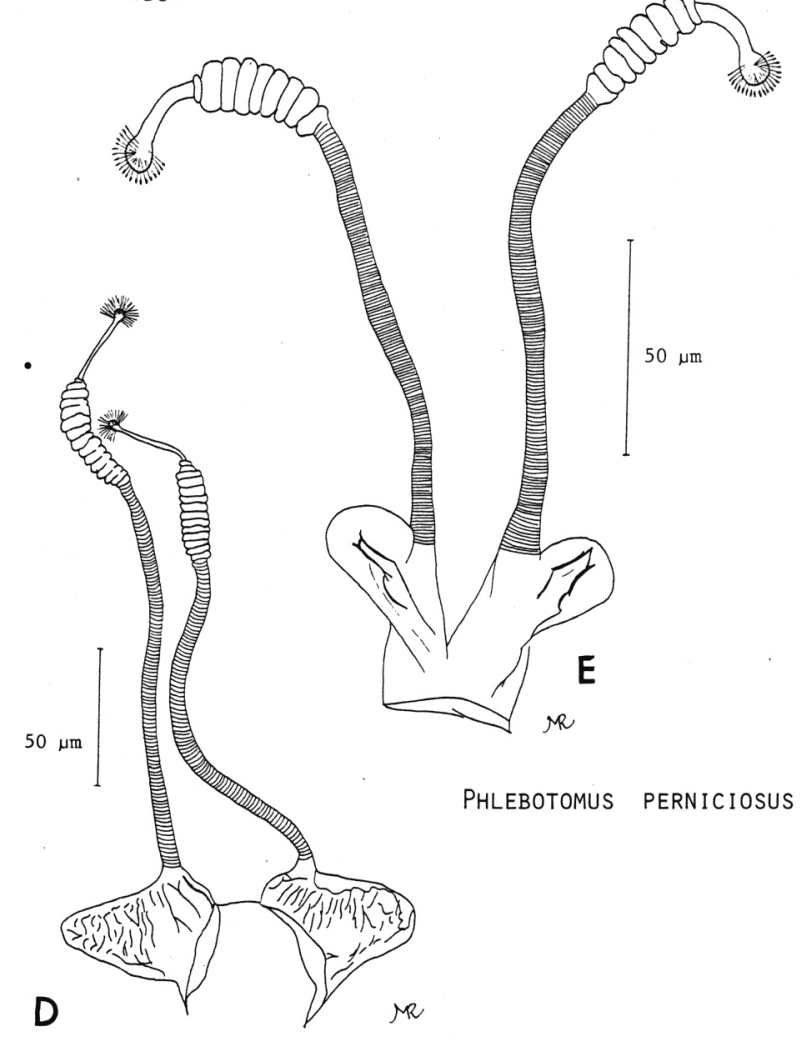

PHLEBOTOMUS PERFILIEWI

FIG. 2. - Spermathèques des femelles de phlébotomes à Gozo (Malte).

par station). 2788 phlébotomes ont été capturés, soit une moyenne de 853 phlébotomes pour 100 papiers, c'est-àdire environ 2,5 fois moins qu'en août 1988 .

L'abondance des différentes espèces est donnée dans le tableau I. Mis à part Sergentomyia minuta (purement herpétophile), Phlebotomus perniciosus est de loin l'espèce dominante.

Le pourcentage des $P$. sergenti est faible aussi bien en 


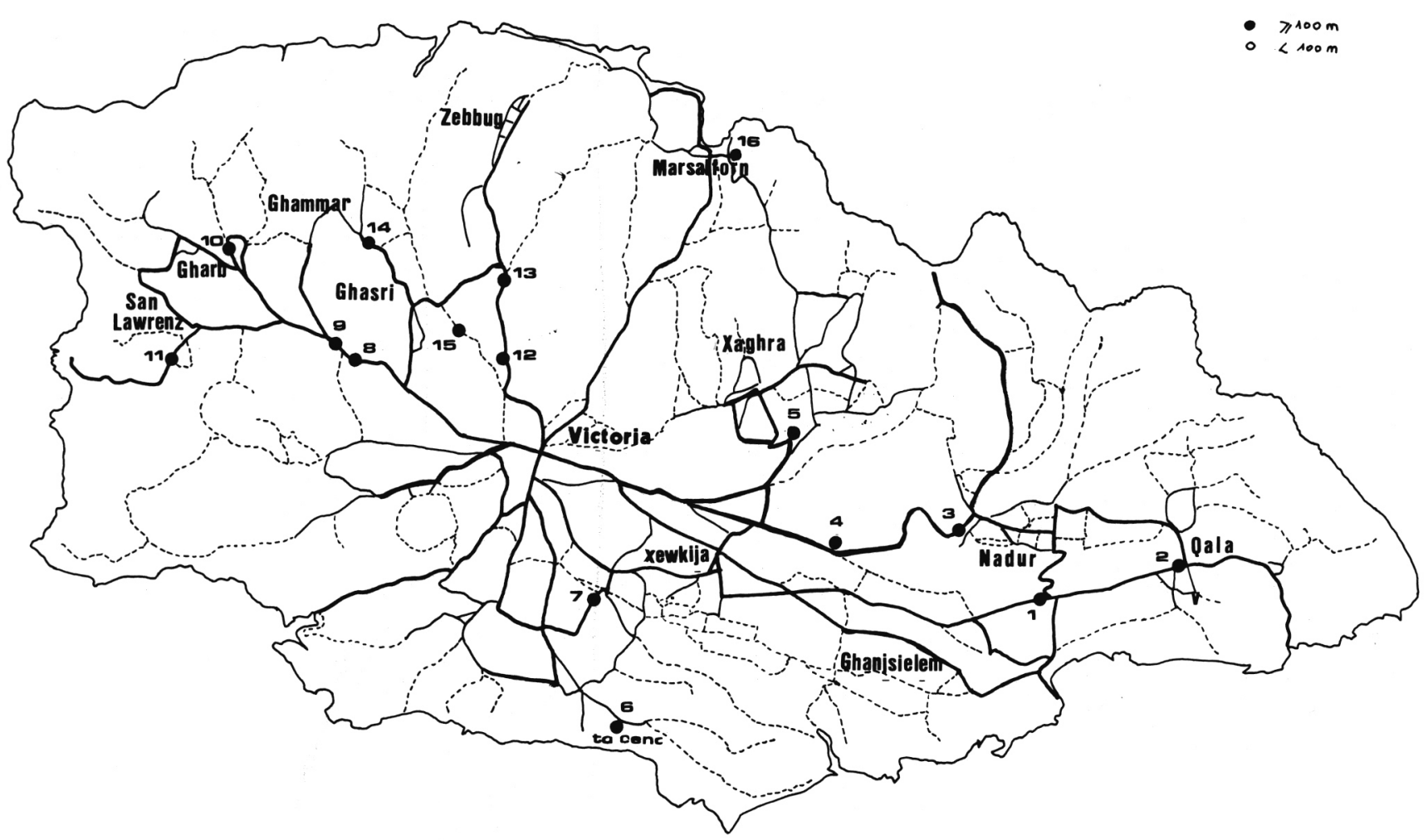

CARTE $N^{\circ} 1$. - Itinéraire transect réalisé en 1988 et 1989 à Gozo (Malte) (au total, 16 stations différentes).

juillet qu'en août, ce qui est un argument en faveur de l'absence de Leishmania tropica à Gozo.

2 - Étude de la répartition des espèces en fonction des biotopes : des pièges lumineux ont été placés dans des endroits qui nous semblaient importants du point de vue épidémiologique :

- à proximité des habitations,

- dans des étables,

- dans des grottes, refuges pour les chiens errants et les rongeurs.

Les résultats des captures figurent dans les tableaux II, III et $I V$.
De l'étude de ces tableaux, il ressort que :

$-P$. perniciosus est partout abondant mais surtout dans les grottes $(95,2 \%)$ où les mâles prédominent et les étables $(87,3 \%)$ où les femelles prédominent ce qui suggère le caractère zoophile des populations locales.

$-P$. perfiliewi, notamment les femelles, est relativement plus abondant autour des habitations (qui ne comportent ni étable, ni poulailler). Il apparaît donc comme nettement anthropophile.

$-P$. sergenti est très peu représenté quel que soit le biotope.

TABlEAU I. - Abondance relative des cinq espèces * (capture par piège adhésif) pour les années 1988 et 1989.

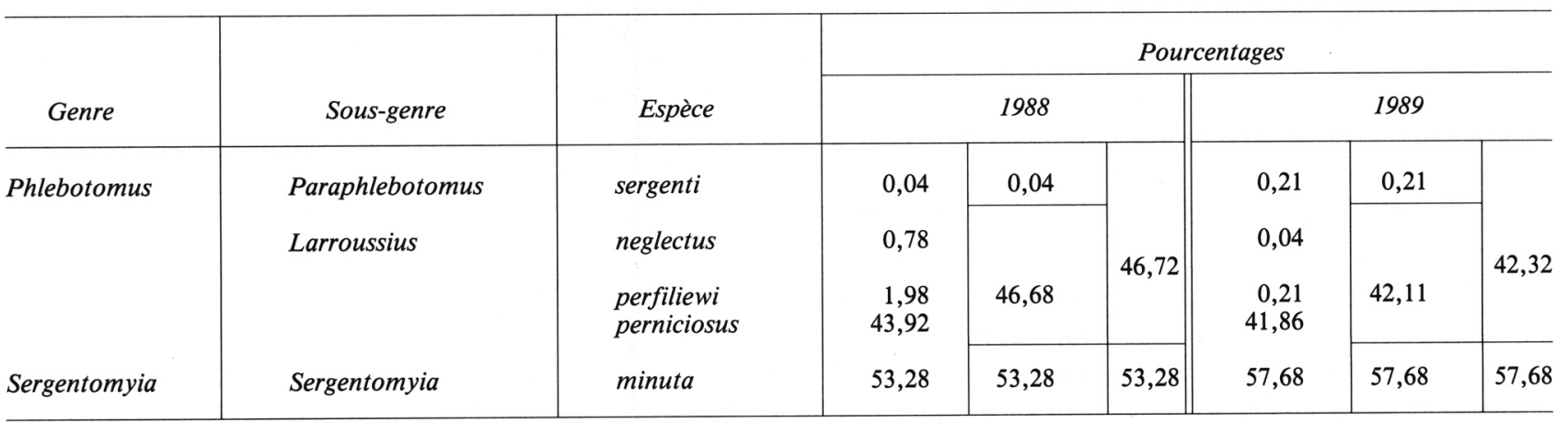

* Aucun P. papatasi n'a été capturé par cette technique. 
TABlEAU II. - Résultats des captures aux pièges lumineux effectuées autour des habitations en août 1988 à Gozo (Malte).

\begin{tabular}{|c|c|c|c|c|c|c|c|c|c|c|c|c|}
\hline \multirow{3}{*}{$\begin{array}{c}\text { Espèces } \\
\text { Nombre par sexe }\end{array}$} & \multicolumn{2}{|c|}{ P. perniciosus } & \multicolumn{2}{|c|}{ P. neglectus } & \multicolumn{2}{|c|}{ P. perfiliewi } & \multicolumn{2}{|c|}{$P$. sergenti } & \multicolumn{2}{|c|}{ S. minuta } & \multirow{2}{*}{\multicolumn{2}{|c|}{ Totaux }} \\
\hline & \multirow{2}{*}{$\begin{array}{l}\sigma^{\prime} \\
53 \\
\end{array}$} & \multirow{2}{*}{$\begin{array}{r}9 \\
38 \\
\end{array}$} & \multirow{2}{*}{$\overbrace{}^{\circ}$} & \multirow{2}{*}{$\begin{array}{l}\text { Q } \\
- \\
-\end{array}$} & \multirow{2}{*}{$\begin{array}{c}0^{\prime} \\
11\end{array}$} & \multirow{2}{*}{$\frac{q}{17}$} & \multirow{2}{*}{$\begin{array}{l}\text { O’ } \\
- \\
-\end{array}$} & \multirow{2}{*}{$\begin{array}{l}q \\
-\end{array}$} & \multirow{2}{*}{$\frac{\sigma^{\prime}}{17}$} & \multirow{2}{*}{$\begin{array}{r}\text { ㅇ } \\
24\end{array}$} & & \\
\hline & & & & & & & & & & & 82 & 79 \\
\hline Pourcentage par sexe & 33,0 & 23,5 & 0,6 & - & 6,8 & 10,6 & - & - & 10,5 & 15,0 & 50,9 & 49,1 \\
\hline $\begin{array}{l}\text { Nombre total par } \\
\text { espèce }\end{array}$ & & & & & & & & & & & & \\
\hline Pourcentage par espèce & & & & & & & & & & & & \\
\hline
\end{tabular}

TABlEAU III. - Résultats des captures aux pièges lumineux effectuées dans des étables en août 1988 à Gozo (Malte).

\begin{tabular}{|c|c|c|c|c|c|c|c|c|c|c|c|c|}
\hline \multirow{3}{*}{$\begin{array}{c}\text { Espèces } \\
\text { Nombre par sexe }\end{array}$} & \multicolumn{2}{|c|}{ P. perniciosus } & \multicolumn{2}{|c|}{ P. neglectus } & \multicolumn{2}{|c|}{ P. perfiliewi } & \multicolumn{2}{|c|}{ P. sergenti } & \multicolumn{2}{|c|}{ S. minuta } & \multirow{2}{*}{\multicolumn{2}{|c|}{ Totaux }} \\
\hline & \multirow{2}{*}{$\begin{array}{r}\sigma^{\prime} \\
66 \\
\end{array}$} & \multirow{2}{*}{$\begin{array}{r}\Upsilon \\
127 \\
\end{array}$} & \multirow{2}{*}{$\begin{array}{l}\sigma^{\prime} \\
- \\
-\end{array}$} & \multirow{2}{*}{$\begin{array}{l}\text { } \\
- \\
-\end{array}$} & \multirow{2}{*}{$\begin{array}{l}\mathrm{O}^{*} \\
1 \\
\end{array}$} & \multirow{2}{*}{$\begin{array}{l}9 \\
4\end{array}$} & \multirow{2}{*}{$\begin{array}{l}O^{\prime} \\
- \\
-\end{array}$} & \multirow{2}{*}{$\begin{array}{l}q \\
2\end{array}$} & \multirow{2}{*}{$\begin{array}{r}\sigma \\
13 \\
\end{array}$} & \multirow{2}{*}{$\frac{q}{8}$} & & \\
\hline & & & & & & & & & & & 80 & 141 \\
\hline Pourcentage par sexe & 29,87 & 57,47 & - & - & 0,45 & 1,81 & - & 0,90 & 5,88 & 3,62 & 36,2 & 63,8 \\
\hline $\begin{array}{r}\text { Nombre total par } \\
\text { espèce }\end{array}$ & & & & & & & & & 21 & & & \\
\hline Pourcentage par espèce & & & & & & & & & & & & \\
\hline
\end{tabular}

TABlEaU IV. - Résultats des captures aux pièges lumineux effectuées dans les grottes en août 1988 à Gozo (Malte).

\begin{tabular}{|c|c|c|c|c|c|c|c|c|c|c|c|c|}
\hline \multirow[b]{2}{*}{ Espèces } & \multicolumn{2}{|c|}{ P. perniciosus } & \multicolumn{2}{|c|}{ P. neglectus } & \multicolumn{2}{|c|}{ P. perfiliewi } & \multicolumn{2}{|c|}{$P$. sergenti } & \multicolumn{2}{|c|}{ S. minuta } & \multirow{2}{*}{\multicolumn{2}{|c|}{ Totaux }} \\
\hline & $\sigma^{*}$ & Q & $\sigma^{\prime}$ & Q & $\sigma^{\prime}$ & q & $\sigma^{\prime}$ & ○ & $\sigma^{\prime}$ & ○ & & \\
\hline Nombre par sexe & 3694 & 993 & 4 & 4 & 19 & 18 & 15 & 2 & 90 & 85 & 3822 & 1102 \\
\hline Pourcentage par sexe & 75,02 & 20,16 & 0,08 & 0,08 & 0,39 & 0,36 & 0,30 & 0,05 & 1,83 & 1,73 & 77,62 & 22,38 \\
\hline $\begin{array}{l}\text { Nombre total par } \\
\text { espèce }\end{array}$ & \multicolumn{2}{|c|}{4687} & \multicolumn{2}{|c|}{8} & \multicolumn{2}{|c|}{37} & \multicolumn{2}{|c|}{17} & \multicolumn{2}{|c|}{175} & \multicolumn{2}{|c|}{4924} \\
\hline Pourcentage par espèce & \multicolumn{2}{|c|}{95,18} & \multicolumn{2}{|c|}{0,16} & \multicolumn{2}{|c|}{0,75} & \multicolumn{2}{|c|}{0,35} & \multicolumn{2}{|c|}{3,56} & \multicolumn{2}{|c|}{-} \\
\hline
\end{tabular}

3 - Étude du pouvoir attractif des petits mammifères :

Des expériences ont été réalisées utilisant la capture aux pièges C. D. C. sans lumière, disposés au-dessus d'appâts animaux.

Trois espèces choisies en fonction de leur grande abondance dans l'île et de leur rôle éventuel en tant que réservoirs de virus ont été expérimentées en parallèle en éliminant toutes les autres causes de variation (disposition des cages par rapport au vent, environnement végétal,...) : le Rat noir (Rattus rattus), le Surmulot (Rattus norvegicus) et le Lapin domestique (Oryctolagus cuniculus).

Le tableau $V$ fait apparaître le pouvoir attractif très marqué du Lapin, les deux espèces de Rats s'étant montrées par ailleurs équivalentes.

3 - ÉtUde PARASTTOLOGIQUe :

Lors des deux missions de 1988 et 1898 , la recherche et l'isolement de souches de Leishmania ont été pratiqués à partir de phlébotomes ainsi qu'à partir de chiens et de rats.

Le typage enzymatique a été confié à L. Gradoni et à M. Gramiccia (Instituto Superiore di Sanita, Rome).

En ce qui concerne les phlébotomes, les résultats des dissections figurent dans le tableau VI. 
TABlEAU V. - Pouvoir attractif du Lapin comparé à celui des Rats.

\begin{tabular}{lcrrr}
\hline \multicolumn{1}{c}{ Espèces } & Rattus sp. & Lapin & Témoin & Total \\
\hline$P$. perniciosus & 281 & 1030 & 136 & 1447 \\
\hline$P$. perfiliewi & 42 & 99 & 28 & 169 \\
\hline$P$. sergenti & - & 1 & - & 1 \\
\hline$P$. neglectus & 1 & 1 & - & 2 \\
\hline S. minuta & 121 & 29 & 67 & 217 \\
\hline
\end{tabular}

$N B$ : les femelles de $P$. sergenti, utilisées pour les élevages, n'ont pas été comptabilisées.

TABleau VI. - Résultats des dissections de phlébotomes.

\begin{tabular}{|c|c|c|c|c|}
\hline & \multicolumn{2}{|c|}{1988} & \multicolumn{2}{|c|}{1989} \\
\hline & \multicolumn{2}{|c|}{ Nombre } & \multicolumn{2}{|c|}{ Nombre } \\
\hline & total & positifs & total & positifs \\
\hline Femelles disséquées & 1463 & 7 & 2063 & 7 \\
\hline P. perniciosus & 1240 & 6 & 1480 & 3 \\
\hline P. perfiliewi & 68 & & 124 & \\
\hline P. neglectus & 7 & & 5 & \\
\hline P. sergenti & 7 & & 21 & \\
\hline P. minuta & 141 & $1 *$ & 433 & $4 *$ \\
\hline
\end{tabular}

* Trypanosomatidae sans doute de reptiles.

\section{ÉTUDE ISOENZYMATIQUE}

DE PHLEBOTOMUS PERNICIOSUS

Dans nos captures, $P$. perniciosus, représente, suivant la technique de piégeage, de 73 à $98 \%$ des Phlebotomus.

Cette espèce, décrite par Newstead en 1911 dans l'île de Malte, a une répartition essentiellement ouest-méditerranéenne, très large : Péninsule Ibérique, France, Italie, Yougoslavie (Dalmatie), Malte, Afrique du Nord, Lybie. Elle a été également signalée à Jersey, en Suisse et en Turquie.

Souvent incriminée dans la transmission de Leishmania infantum, son efficacité en tant que vecteur de même que son éthologie varient d'une région à l'autre.

En outre, il existe plusieurs espèces voisines ( $P$. tobbi, $P$. longicuspis, $P$. langeroni) qui ont souvent été décrites à l'origine comme des sous-espèces ou des "variétés » et dont l'individualisation peut prêter à discussion.

C'est pourquoi il nous a paru intéressant de comparer nos populations de Gozo avec des populations d'origines géographiques différentes :

- Espagne (Murcia), exemplaires provenant d'un élevage entretenu au laboratoire par M. et R. Killick-Kendrick,

- France (Nice) : captures manuelles,

- Italie (Bologne) : captures manuelles.

Tous ces $P$. pernicious ont été congelés immédiatement après leur capture et sont conservés dans l'azote liquide.

Deux méthodes d'analyse enzymatique ont été utilisées :

- électrophorèse en gel d'amidon selon la technique de Pasteur et coll. (1987) adaptée par Voltz-Kristensen (1987),

TABLEAU VII. - Zymogrammes et génotypes de la phosphoglucomutase de P. perniciosus d'origines géographiques différentes.

\begin{tabular}{|c|c|c|c|c|c|c|c|c|c|}
\hline \multirow{5}{*}{$\begin{array}{r}5,85 \\
\text { Zymogrammes } \\
5,54 \\
5,20 \\
5,06+\end{array}$} & \multicolumn{9}{|l|}{$p \mathrm{H}$} \\
\hline & & & & & & & - & & $\mathrm{F}$ \\
\hline & & & & & & & & & D \\
\hline & & & & & & & & & $\mathrm{C}$ \\
\hline & 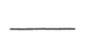 & 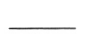 & & & & & & & $\begin{array}{l}\text { B } \\
\text { A }\end{array}$ \\
\hline $\begin{array}{r}\text { Génotypes } \\
\text { observés }\end{array}$ & $\mathrm{A} / / \mathrm{C}$ & $\mathrm{A} / / \mathrm{D}$ & $\mathrm{B} / / \mathrm{B}$ & $\mathrm{B} / / \mathrm{D}$ & $\mathrm{D} / / \mathrm{D}$ & $\mathrm{D} / / \mathrm{E}$ & $\mathrm{D} / / \mathrm{F}$ & $\mathrm{E} / / \mathrm{E}$ & \\
\hline Origines & & & & & & & & & \\
\hline $\begin{array}{l}\text { Gozo (Malte) } \\
\text { (population naturelle) }\end{array}$ & 2 & 4 & 0 & 16 & 213 & 8 & 1 & 2 & 246 \\
\hline $\begin{array}{l}\text { Bologne (Italie) } \\
\text { (population naturelle) }\end{array}$ & 0 & 0 & 0 & 1 & 41 & 0 & 0 & 0 & 42 \\
\hline $\begin{array}{l}\text { Nice (France) } \\
\text { (population naturelle) }\end{array}$ & 0 & 0 & 1 & 1 & 12 & 0 & 0 & 0 & 14 \\
\hline $\begin{array}{l}\text { Murcia (Espagne) } \\
\text { (élevage) }\end{array}$ & 0 & 0 & 0 & 0 & 7 & 0 & 0 & 0 & 7 \\
\hline
\end{tabular}


TABLEAU VIII. - Fréquences alléliques de la phosphoglucomutase de P. perniciosus d'origines géographiques différentes.

\begin{tabular}{lcccccc}
\hline Origines & $\begin{array}{c}\text { Nombre de } \\
\text { phlébotomes }\end{array}$ & $A$ & $B$ & $C$ & $E$ & 0,0020 \\
\hline Gozo & 246 & 0,0122 & 0,0325 & 0,0041 & 0,9247 & 0,0244 \\
\hline Bologne & 42 & 0 & 0,0119 & 0 & 0,9881 & 0 \\
\hline Nice & 14 & 0 & 0,1701 & 0 & 0,8929 & 0 \\
\hline Murcia & 7 & 0 & 0 & 0 & 1 & 0 \\
\hline
\end{tabular}

— isoélectrofocalisation (I.E.F.) en gel d'agarose selon la technique décrite par Voltz-Kristensen (1987).

Dans un premier temps, huit enzymes ont été ainsi étudiées : estérase (EST-E.C.3.1.1.1.), hexokinase(HKE.C.2.7.1.1.), lactate déshydrogénase (LDH-E.E.1.1.1.27), malate déshydrogénase (MDH-E.C.1.1.1.37), enzyme malique (ME-E.C.1.1.1.40), phosphoglucomutase (PGME.C.2.7.5.1.), phospho-glucoisomérase (PGI-E.C.5.3.1.9.) et super oxyde dismutase (SOD-E.C.1.15.1.1.).

Par la suite nous n'avons retenu que trois enzymes (PGI, PGM et HK) et une seule technique : l'I.E.F.

Au total, $553 P$. perniciosus, toutes origines confondues, ont été étudiés dont 56 testés en "double spot " (révélation de deux enzymes à partir d'un même phlébotome), ce qui permet de mettre en évidence l'indépendance des gênes codant ces enzymes.

\section{$a$ - PGM (tableaux VII et VIII)}

L'observation des phénotypes hétérozygotes permet de définir le type structural de l'enzyme étudiée (monomérique, dimérique ou polymérique) ainsi que le nombre de locus codant pour cette enzyme (Tibayrenc, 1979). Pour la PGM, la présence d'individus sauvages à 1 ou 2 bandes conduit à l'hypothèse suivante : la PGM est un monomère et pourrait être codée par six allèles (désigné ici de $\mathrm{A}$ à $\mathrm{F}$ ) pour un locus. Ceci est une hypothèse, qui ne prend pas en compte l'existence d'allèle muet dont on ne peut exclure la présence.

Les résultats du tableau VII montrent un net polymorphisme de la PGM pour les phlébotomes de Gozo. Le génotype D/D est le plus représenté dans les quatre échantillons étudiés. La PGM ne semble pas constituer un bon marqueur des populations pour $P$. perniciosus contrairement à ce qui est observé chez $P$. perfiliewi perfiliewi par Ward et coll. (1981).

\section{$b$ - PGI (tableaux IX et $X$ )}

Trois phénotypes différents sont observés pour les populations de Gozo, Nice et Murcia. Dans la population de Gozo, $139 P$. perniciosus présentent un phénotype à une bande de migration et 7 un zymogramme à trois bandes dont une des bandes extrêmes est de mobilité identique à celle du phénotype homozygote et la bande intermédiaire est de forte activité enzymatique. L'autre phénotype homozygote a été observé dans la population de Murcia une seule fois.
La présence de ces hétérozygotes à trois bandes suggère que les isoenzymes de la PGI ont une structure dimérique et sont codées par un seul locus (nous avons observé un seul individu non hétérozygote présentant deux bandes, ceci pourrait peut-être correspondre à un deuxième locus (Ward et coll., 1981), ou encore à la trace d'un repas sanguin ou à la présence d'un parasite).

D'autre part, en testant la PGM et la PGI sur un même exemplaire, on se rend compte que ces deux enzymes sont indépendantes (lorsqu'il y a hétérozygotie pour une enzyme, on ne la retrouve pas obligatoirement pour l'autre enzyme).

\section{c-H. K. (tableaux IX et XII)}

La population de Gozo est plus hétérogène pour l'hexokinase que celle de France ou d'Espagne où seulement deux allèles sont retrouvés : $B$ et $C$. Ceci est en partie dû au

TABLEAU IX. - Zymogrammes et génotypes de la phosphoglucoisomérase de $\mathrm{P}$. perniciosus d'origine géographique différente.

\begin{tabular}{|c|c|c|c|c|c|c|}
\hline \multirow{2}{*}{ Zymogrammes } & $5,85^{-}$ & $p \mathrm{H}$ & & & B & \\
\hline & 5,20 & & - & $=$ & A & Total \\
\hline Origines & Génotypes & observés & $A / / A$ & $A / / B$ & $B / / B$ & \\
\hline
\end{tabular}

\begin{tabular}{lcccc}
\hline Gozo (Malte) & 139 & 7 & 0 & 146 \\
\hline Nice (France) & 20 & 0 & 0 & 20 \\
\hline Murcia (Espagne) & 14 & 6 & 1 & 21 \\
\hline
\end{tabular}

TABLEAU X. - Fréquences alléliques de la phosphoglucoisomérase de $\mathrm{P}$. perniciosus d'origine géographique différente.

\begin{tabular}{lcll}
\hline \multicolumn{1}{c}{ Origines } & $\begin{array}{c}\text { Nombre de } \\
\text { phlébotomes }\end{array}$ & $A$ & $B$ \\
\hline Gozo (Malte) & 146 & 0,976 & 0,024 \\
\hline Nice (France) & 20 & 1 & 0 \\
\hline Murcia (Espagne) & 21 & 0,8095 & 0,1905 \\
\hline
\end{tabular}


TABLEAU XI. - Zymogrammes et génotypes de l'hexokinase de P. perniciosus d'origines géographiques différentes.

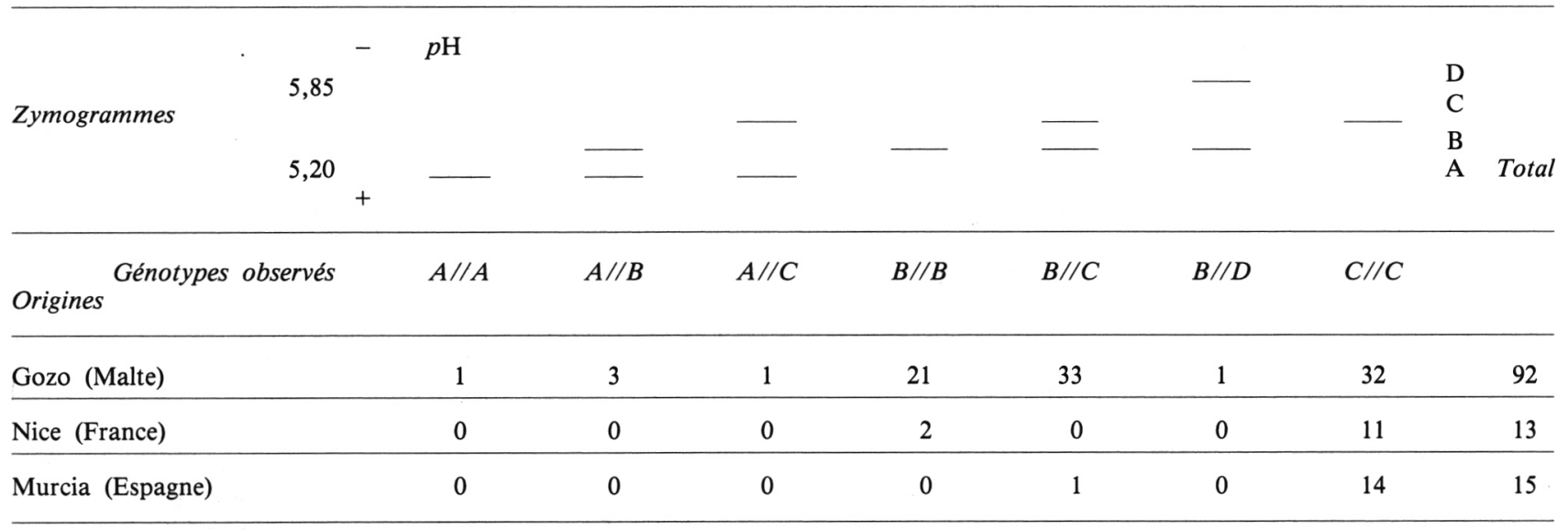

TABLEAU XII. - Fréquences alléliques de l'hexokinase de $\mathrm{P}$. perniciosus d'origines géographiques différentes.

\begin{tabular}{lccccc}
\hline Origines & $\begin{array}{c}\text { Nombre de } \\
\text { phlébotomes }\end{array}$ & $A$ & $B$ & $C$ & $D$ \\
\hline Gozo & 92 & 0,0326 & 0,4293 & 0,5326 & 0,0055 \\
\hline Nice & 13 & 0 & 0,1538 & 0,8462 & 0 \\
\hline Murcia & 15 & 0 & 0,0333 & 0,9667 & 0 \\
\hline
\end{tabular}

faible échantillonnage testé dans ces deux populations dont une (Murcia) est une population d'élevage. Cependant, l'hexokinase se révèle un bon marqueur des populations de $P$. perniciosus, comme Ward et coll. (1981) l'observent sur leurs captures de France et de Tunisie.

Des sept électromorphes mis en évidence, on peut déduire l'existence de quatre allèles. Les hétérozygotes à deux bandes suggèrent que l'hexokinase est monomérique. Par ailleurs, la PGM et la HK sont indépendantes.

Cette première analyse isoenzymatique de $P$. perniciosus de Gozo, ainsi que les résultats déjà obtenus à Corfou (Voltz-Kristensen et coll., à paraître) permettent d'envisager l'application de cette méthode à l'étude génétique des populations de Larroussius dans les foyers méditerranéens de leishmaniose.

\section{RÉFÉRENCES}

Adler S., Théodor O.: Investigations on mediterranean Kala Azar. VIII Further observations on mediterranean sandflies. Proc. Roy. Soc. (B), 1935, 116, 505-515.

Busuttil A.: Kala-Azar in the Maltese Islands. Trans. R. Soc. Trop. Med. Hyg., 1974, 68, 236-240.

Cachia E., Fenerh F.: A review of Kala-azar in Malta from 1947 to 1962. Trans. R. Soc. Trop. Med. Hyg., 1964, 58, 234-241.

Croset H., Rioux J. A., Léger N., Houin R., Cadi-Soussi M.,
Benmansour N., Maistre M. : Les méthodes d'échantillonnage des populations de phlébotomes en région méditerranéenne. Coll. Internat. C.N.R.S., 1977, $\mathrm{n}^{\circ} 239,139-151$.

Léger N., Pesson B., Madulo-Leblond G., Abonnenc E. : Sur la différenciation des femelles du sous-genre Larroussius Nitzulescu, 1931 (Diptera, Phlebotomidae) de la région méditerranéenne. Ann. Parasitol. Hum. Comp., 1983, 58, 611-623.

Marett P. J.: The Phlebotomus flies of the Maltese Islands. $J$. Roy. Army. Med. Corps, 1913, 20, 162-171.

Ministry of Health: Leishmaniasis reported cases in Malta and Gozo over recent years. Document confidentiel, Malta, 19 avril 1989.

Newstead R.: The papataci flies (Phlebotomus) of the Maltese Islands. Bull. Ent. Res., 1911, 2, 47-48.

Newstead R.: Notes on Phlebotomus with descriptions of new species. Part.II. Bull. Ent. Res., 1914, 5,179-192.

Pasteur N., Pasteur G., Bonhomme F., Catalan J., BrittonDavidian J. : Manuel technique de génétique par électrophorèse des protéines. Lavoisier, Technique et Documentation, Paris, 1987, $217 \mathrm{p}$.

Peters W., Killick-Kendrick R.: The leishmaniases in biology and medecine. Academic Press, London, 1987, 951 pp.

Rioux J. A., Lanotte G., Maazoun R., Perello R., Pratlong F. : Leishmania infantum Nicolle, 1908, agent du bouton d'Orient autochtone. A propos de l'identification biochimique de deux souches isolées dans les Pyrénées Orientales. C.R. Acad. Sc. Paris, 1980, 291, Série D.701.

Tibayrenc M. : Les isoenzymes et l'entomologie médicale. Cah. ORSTOM, Ser. Ent. Med. Parasitol., 1979, 17, 249-256.

Théodor O.: Transmission of Kala-azar in Malta. Trans. R. Soc. Trop. Med. Hyg., 1976, 70, 259-260.

Vella Briffa D.: Cutaneous leishmaniasis in the Maltese Islands (Letter). Br. J. Dermatol., 1985, 13, 370-371.

Voltz-Kristensen A. : Contribution à l'identification par la biométrie et le typage enzymatique de souches expérimentales et de populations naturelles de Trématodes du genre Echinostoma. Th. Pharm. (ès Sc. Pharm.), 1987, 202 p.

Ward R. D., Bettini S., Maroli M., McGarry J. W., Draper A.: Phosphoglucomutase polymorphism in Phlebotomus perfiliewi perfiliewi Parrot (Diptera : Psychodidae) from central and northern Italy. Ann. Trop. Med. Parasitol., 1981, 75, 653-661.

Ward R. D., Pasteur N., Rioux J. A.: Electrophoretic studies on genetic polymorphism and differenciation of phlebotomine sandflies (Diptera : Psychodidae) from France and Tunisia. Ann. Trop. Med. Parasitol., 1981, 75, 235-245.

(C) Masson, Paris 1991 